\title{
Successful treatment of lower urinary tract infections with oral fosfomycin: a report of three cases
}

\author{
Juliana Oliveira da Silva ${ }^{[1]}$, Maria Cecilia Zorat Yu${ }^{[1]}$, \\ André Do i[1],[2], Maria Rita Elmor de Araujo ${ }^{[2]}$ \\ Pedro Aurélio Mathiasi Neto ${ }^{[2]}$ and Guilherme Henrique Campos Furtado ${ }^{[1],[2]}$
}

[1]. Setor de Doenças Infecciosas, Hospital São Paulo, Universidade Federal de São Paulo, São Paulo, Brasil. [2]. Hospital do Coração, São Paulo, São Paulo, Brasil.

\begin{abstract}
Infections due to multidrug-resistant organisms continue to increase, and therapeutic options remain scarce. Given this challenge, it has become necessary to use older antimicrobials for treatment of these pathogens. We report three patients with lower urinary tract infections caused by Klebsiella pneumoniae carbapenemase (KPC)-producing Klebsiella pneumoniae who were successfully treated with a seven-day course of oral fosfomycin monotherapy.
\end{abstract}

Keywords: Fosfomycin. Urinary tract infection treatment. KPC-producing Klebsiella pneumoniae.

\section{INTRODUCTION}

The emergence of multidrug-resistant (MDR), mainly carbapenem-resistant, Klebsiella pneumoniae has prompted reevaluation of nontraditional antibiotics, such as fosfomycin, due to their efficacy for the treatment of both urinary and systemic infections. In particular, data regarding the use of fosfomycin for the treatment of infections caused by contemporary MDR gram-negative pathogens are warranted to further evaluate the optimal clinical use of this revived antimicrobial agent ${ }^{(1)}$.

Studies supporting the use of this drug as monotherapy for these infections are limited. Here, we describe three patients with lower urinary tract infections (UTIs) caused by Klebsiella pneumoniae carbapenemase (KPC)-producing $K$. pneumoniae who were successfully treated with oral fosfomycin monotherapy.

\section{CASE REPORT}

The first case was a 70-year-old man who underwent coronary bypass surgery, without complications. Thirteen days after the surgery, the patient presented with dysuria and urgency without fever. A urinary tract infection was diagnosed. The urine

Corresponding Author: Dra. Juliana Oliveira da Silva. Setor de Doenças Infecciosas/Hospital São Paulo/UNIFESP. Rua Napoleão de Barros 690/2 ${ }^{\circ}$ andar, 04024-000 São Paulo, Brasil.

Phone: 5511 5576-4463

e-mail: juoliveira_silva@yahoo.com.br

Received 26 November 2014

Accepted 16 March 2015 analysis showed 750,000 leukocytes $/ \mathrm{mL}$, and the culture yielded KPC-producing K. pneumoniae.

The second case was a 48-year-old woman who underwent cardiac valve surgery owing to severe dysfunction of her existing metallic mitral valve. Because of surgical complications, she was admitted to the intensive care unit (ICU) with cardiogenic shock and severe ventricular dysfunction. She remained in the ICU for 35 days. Eight days post-discharge, the patient presented with dysuria and pelvic discomfort. The urine analysis showed 733,000 leukocytes/ $\mathrm{mL}$, and the culture yielded KPC-producing K. pneumoniae.

The third case was an 80-year-old man with chronic renal failure on peritoneal dialysis who was admitted to the hospital with a lower UTI. The urine analysis showed 265,000 leukocytes $/ \mathrm{mL}$, and the culture yielded KPC-producing K. pneumoniae.

A urinary catheter was not used by any of the three patients. Treatment for all patients included $3 \mathrm{~g}$ oral fosfomycin every $12 \mathrm{~h}$ for seven days, without adverse effects. Urine analyses were conducted, and cultures were collected at the end of the treatment and again one week later. No leukocyturia was evident, and all cultures remained negative.

Upon admission, the urine cultures of all three patients showed colony counts $>100,000 \mathrm{CFU} / \mathrm{mL}$ (colony-forming units per milliliter), with growth of $K$. pneumoniae identified at $>95 \%$ probability using the Vitek 2 Compact ${ }^{\circledR}$ (bioMerieux) automated system. The modified Hodge test and polymerase chain reaction for gene $b l a_{\mathrm{KPC}}$ were positive for all isolates.

A disk diffusion test was performed using discs with $200 \mu \mathrm{g}$ fosfomycin trometamol supplemented with $50 \mu \mathrm{g}$ glucose-6-phosphate. The minimum inhibitory concentrations (MICs) were determined using the agar dilution method according to the Clinical and Laboratory Standards Institute (CLSI) recommendations. Briefly, Mueller-Hinton agar plates 
were supplemented with $100 \mu \mathrm{L}$ glucose-6-phosphate at a concentration of $5 \mathrm{mg} / \mathrm{mL}$. Fosfomycin concentrations ranged from $0.125 \mu \mathrm{g} / \mathrm{mL}$ to $256 \mu \mathrm{g} / \mathrm{mL}$. A drug-free plate was used for growth control, and the standard strains Escherichia coli ATCC 25922 and Pseudomonas aeruginosa ATCC 27853 were used as quality controls. The results were within the expected ranges, for both methodologies. For agar dilution, the three isolates as well as the controls were used to prepare a solution with a turbidity of $0.5 \mathrm{McF}$ arland (approximately $10^{8} \mathrm{CFU} / \mathrm{mL}$ ). This inoculum was diluted to $1: 10$ for a concentration of $10^{7} \mathrm{CFU} /$ $\mathrm{mL}$. A $2-\mu \mathrm{L}$ aliquot was inoculated on the plates to yield a final inoculum of $10^{4} \mathrm{CFU} / \mathrm{mL}$. The plates were incubated at $35^{\circ} \mathrm{C}$ and read after $18 \mathrm{~h}$.

As criteria to evaluate the susceptibility of $K$. pneumoniae to fosfomycin in the current CLSI guidelines were not available, the results were interpreted according to the breakpoints used for $E$. coli in UTIs: resistant, $\leq 12 \mathrm{~mm}$; intermediate, 13 $15 \mathrm{~mm}$; susceptible: $\geq 16 \mathrm{~mm}$ for disc diffusion and susceptible, MIC $\leq 64 \mu \mathrm{g} / \mathrm{mL}$; intermediate, MIC $128 \mu \mathrm{g} / \mathrm{mL} ;$ resistant,

TABLE 1 - Comparative results of disk diffusion and agar dilution for three isolates.

\begin{tabular}{lcc}
\hline Strain & DD $(\mathrm{mm})$ & MIC $(\mu \mathrm{g} / \mathrm{mL})$ \\
\hline Patient 1 & $19(\mathrm{~S})$ & $128(\mathrm{I})$ \\
Patient 2 & $13(\mathrm{I})$ & $128(\mathrm{I})$ \\
Patient 3 & $22(\mathrm{~S})$ & $8(\mathrm{~S})$ \\
Escherichia coli & $23(\mathrm{~S})$ & $1(\mathrm{~S})$ \\
Pseudomonas aeruginosa & - & $4(\mathrm{~S})$ \\
\hline
\end{tabular}

DD: disk diffusion; MIC: minimum inhibitory concentration; S: susceptible; I: intermediate.

TABLE 2 - Minimum inhibitory concentration results for other antimicrobial agents using the Vitek 2 automated method.

\begin{tabular}{lccc}
\hline & Patient 1 & Patient 2 & Patient 3 \\
\hline Amikacin & $4(\mathrm{~S})$ & $4(\mathrm{~S})$ & $4(\mathrm{~S})$ \\
Cefepime & $\geq 64(\mathrm{R})$ & $\geq 64(\mathrm{R})$ & $\geq 64(\mathrm{R})$ \\
Cefotaxime & $\geq 64(\mathrm{R})$ & $\geq 64(\mathrm{R})$ & $\geq 64(\mathrm{R})$ \\
Ceftazidime & $\geq 64(\mathrm{R})$ & $\geq 64(\mathrm{R})$ & $\geq 64(\mathrm{R})$ \\
Ciprofloxacin & $\geq 4(\mathrm{R})$ & $\geq 4(\mathrm{R})$ & $\geq 64(\mathrm{R})$ \\
Colistin & $\leq 0.5(\mathrm{~S})$ & $\leq 0.5(\mathrm{~S})$ & $\leq 0.5(\mathrm{~S})$ \\
Ertapenem & $\geq 8(\mathrm{R})$ & $\geq 8(\mathrm{R})$ & $\geq 8(\mathrm{R})$ \\
Gentamicin & $\leq 1(\mathrm{~S})$ & $\leq 1(\mathrm{~S})$ & $\leq 1(\mathrm{~S})$ \\
Imipenem & $4(\mathrm{R})$ & $\geq 16(\mathrm{R})$ & $\geq 16(\mathrm{R})$ \\
Meropenem & $1(\mathrm{~S})$ & $\geq 16(\mathrm{R})$ & $2(\mathrm{I})$ \\
Piperacillin-tazobactam & $\geq 128(\mathrm{R})$ & $\geq 128(\mathrm{R})$ & $\geq 128(\mathrm{R})$ \\
\hline
\end{tabular}

S: susceptible; R: resistant; I: intermediate.
MIC $\geq 256 \mu \mathrm{g} / \mathrm{mL}$ for agar dilution ${ }^{(2)}$. These breakpoints have been used previously in similar studies ${ }^{(1)(3)(4)(5)(6)}$.

The susceptibility test results for fosfomycin are shown in Table 1. Only one minor error for the first isolate (agar dilution in the intermediate category and disk diffusion zone indicating susceptible, but near the intermediate zone) was found, reflecting good concordance between both methodologies. Other antimicrobial agents were also tested using Vitek 2 Compact ${ }^{\circledR}$, and the MIC results are shown in Table 2. High levels of resistance to cephalosporins, quinolones, and piperacillin/ tazobactam and susceptibility to aminoglycosides and colistin were found. MICs to carbapenems varied, although all isolates were carbapenemase producers.

\section{DISCUSSION}

Therapeutic options against infections caused by KPCproducing $K$. pneumoniae are often limited ${ }^{(5)}$. Thus, new therapeutic strategies against these isolates are urgently needed ${ }^{(7)(8)}$. Oral fosfomycin is an old antimicrobial drug marketed in Brazil, the United States, and other countries as a single dose for the treatment of uncomplicated UTIs. It shows potent bactericidal action against many gram-negative and gram-positive pathogens ${ }^{(6)}$. Unfortunately, the parenteral disodium salt of fosfomycin is not yet available in Brazil $^{(7)(9)}$.

Although fosfomycin tromethamine is only indicated for lower UTIs, it has been studied in both uncomplicated and complicated lower UTIs caused by MDR uropathogens because it shows synergistic action with other antimicrobial drugs ${ }^{(10)(11)}$. The parenteral formulation has been used in combination therapy to treat a wide range of infections including pneumonia and septicemia with cure rates $>80 \%{ }^{(12)}$.

Given its low rates of resistance and the available evidence, fosfomycin may serve as a useful option for oral treatment of MDR uropathogens ${ }^{(1)}$. Surprisingly, data regarding in vitro and in vivo activity of fosfomycin against KPC-producing $K$. pneumoniae isolates have been lacking until recently. A better understanding of the most appropriate oral dosing regimen against MDR uropathogens that cause UTIs is needed ${ }^{(8)}$.

Neuner et al. described the microbiological outcomes of treatment with oral fosfomycin monotherapy for UTIs caused by MDR pathogens, including 13 carbapenem-resistant $K$. pneumoniae isolates. Patients received an average of $2.9 \pm 1.8$ doses (each dose $=3 \mathrm{~g}$ fosfomycin tromethamine) per course of therapy. A $92 \%$ in vitro fosfomycin susceptibility was observed with a corresponding microbiological cure of $46 \%$ (6/13 patients). Most patients who had treatment failure were immunosuppressed or had ureteral stents suggesting caution in using fosfomycin monotherapy in these types of patients ${ }^{(1)}$.

In the present study, we analyzed the susceptibility of three samples using disk diffusion and agar dilution. Only the isolate from patient 3 was susceptible to fosfomycin by both methods. The other two isolates were in the intermediate category in one or both methodologies. Even so, all patients experienced a microbiological and clinical cure with the applied dose regimen. 
The presence of a urinary catheter is considered a complicating factor that may be related with microbiological failure, probably due to the biofilm structure that gram-negative bacteria can produce. Thus, the absence of a catheter might have contributed to the successful treatment of these three patients. Furthermore, fosfomycin in combination with other antibiotics, e.g., colistin, gentamicin, or carbapenems, might be able to eradicate biofilms caused by gram-negative bacteria ${ }^{(1)}$.

The incidence of KPC-related UTIs was very low in the hospital during the study period, with approximately $4.5 \%$ of urine cultures positive for KCP during the 1-year period. These three cases were the first three patients diagnosed with a KPC-related UTI and the only cases who were treated with fosfomycin during this period. The major therapeutic options for patients with a KPC-related UTI were polymyxins (polymyxin $\mathrm{B}$ or colistin) or aminoglycosides combined with carbapenems. We opted to treat these patients with fosfomycin because they had less severe UTIs and did not have a urinary indwelling catheter. Owing to the high probability of the development of resistance, we have not utilized fosfomycin as monotherapy for uncomplicated KPC-related UTIs in recent years.

In conclusion, fosfomycin demonstrated reasonable in vitro activity against contemporary KPC-producing $K$. pneumoniae isolates, representing a possible alternative to other antimicrobials.

Despite the small number of cases (three), our results demonstrated that oral fosfomycin is a possible therapeutic option for lower UTIs caused by KPC-producing K. pneumoniae isolates.

\section{REFERENCES}

1. Neuner EA, Sekeres J, Hall GS, van Duin D. Experience with fosfomycin for treatment of urinary tract infections due to multidrug-resistant organisms. Antimicrob Agents Chemother 2012; 56:5744-5748.

2. Wayne PA. Clinical and Laboratory Standards Institute (CLSI). Performance Standards for Antimicrobial Susceptibility Testing. $21^{\text {th }}$ informational supplement. CLSI document M100-S21. CLSI; 2011.

3. Falagas ME, Giannopoulou KP, Kokolakis GN, Rafailidis PI. Fosfomycin: use beyond urinary tract and gastrointestinal infections. Clin Infect Dis 2008; 46:1069-1077.

4. Falagas ME, Kastoris AC, Karageorgopoulos DE, Rafailidis PI. Fosfomycin for the treatment of infections caused by multidrugresistant non-fermenting Gram-negative bacilli: a systematic review of microbiological, animal and clinical studies. Int $\mathrm{J}$ Antimicrob Agents 2009; 34:111-120.

5. Keating GM. Fosofomycin trometamol: a review of its use as a single-dose oral treatment for patients with acute lower urinary tract infections and pregnant women with asymptomatic bacteriuria. Drugs 2013; 73:1951-1966.

6. Maraki S, Samonis G, Rafailidis PI, Vouloumanou EK, Mavromanolakis E, Falagas ME. Susceptibility of urinary tract bacteria to fosfomycin. Antimicrob Agents Chemother 2009; 53:4508-4510.

7. Endimiani A, Patel G, Hujer KM, Swaminathan M, Perez F, Rice $\mathrm{LB}$, et al. In vitro activity of fosfomycin against blaKPC-containing Klebsiella pneumoniae isolates, including those nonsusceptible to tigecycline and/or colistin. Antimicrob Agents Chemother 2010; 54:526-529.

8. de Cueto M, Lopez L, Hernandez JR, Morillo C, Pascual A. In vitro activity of fosfomycin against extended-spectrum- $\beta$ lactamase-producing Escherichia coli and Klebsiella pneumoniae: comparison of susceptibility testing procedures. Antimicrob Agents Chemother 2006; 50:368-370.

9. Lopez-Cerero L, de Cueto M, Diaz-Guerrero MA, Morillo C, Pascual A. Evaluation of the Etest method for fosfomycin susceptibility of ESBL-producing Klebsiella pneumoniae. J Antimicrob Chemother 2007; 59:810-812.

10. Schito GC, Naber KG, Botto H, Palou J, Mazzei T, Gualco L, et al. The ARESC study: an international survey on the antimicrobial resistance of pathogens involved in uncomplicated urinary tract infections. Int J Antimicrob Agents 2009; 34:407-413.

11. Popovic M, Steinort D, Pillai S, Joukhadar C. Fosfomycin: an old, new friend? Eur J Clin Microbiol Infect Dis 2010; 29:127-142.

12. Roussos N, Karageorgopoulos DE, Samonis G, Falagas ME. Clinical significance of the pharmacokinetic and pharmacodynamic characteristics of fosfomycin for the treatment of patients with systemic infections. Int J Antimicrob Agents 2009; 34:506-515. 